\title{
GLI ACCORDI DI BASILEA, IL RATING TRA BANCHE E IMPRESE
}

\author{
AGREMENTS BASEL, RATING BETWEEN BANKS AND ENTERPRISES
}

\author{
Alfio Leotta \\ Università di Catania - Sicília - Itália
}

\begin{abstract}
Sommario: Ricerca sul rapporto nel sistema economico internazionale tra le banche e le imprese e la loro cooperazione integrata e asimmetrica partendo dagli assunti degli accordi di Basilea, Comitato istituito appunto per la vigilanza bancaria, e come questi accordi hanno influito sui processi economici dell'Europa in genere e dell'Italia in particolare. Scopo principale di questa indagine è: instituire una ricerca su tutti gli organi comunitari che sono stati promotori di rating ed hanno favorito il rapporto fra banche e imprese.
\end{abstract}

Parole chiave: Acordi di Basile; Sistema Economico Internazionale; Vigilanza Bancaria.

Abstract: Research on the relationship in the international economic system between banks and enterprises and their cooperation and integrated asymmetrical starting from the assumptions of the Basel Accords, the Committee established precisely on Banking Supervision, and as these agreements have affected the economic processes of Europe in general and Italy in particular. The main purpose of this survey is: to institute a search of all the bodies that were promoters of rating and fostered the relationship between banks and enterprises.

Keywords: Basel Accords; International Economic System; Banking Supervision.

\section{Introduzione}

Gli accordi di Basilea in genere identificano il lavoro del Comitato di Basilea per la vigilanza bancaria, finalizzato all'aggiornamento della normativa internazionale riguardante i requisiti patrimoniali delle banche. Soffermandoci soprattutto sulla più recente normativa proposta da questo Comitato, chiamato Basilea3, si tratta di chiarire quali trasformazioni questa potrà apportare nei prossimi anni e la rilevanza di questi cambiamenti per le imprese, e per l'occupazione in genere, che devono confrontarsi con le recenti profonde innovazioni nel contesto macroeconomico, subendo le conseguenze 
congiunturali di una realtà economica in radicale cambiamento. Ci soffermeremo quindi, sull'importanza delle regole di Basilea per ogni attore della scena economica sia esso una banca, un'impresa, uno Stato od un piccolo investitore, oppure lo stesso lavoratore. Ha in sé un'importanza sostanziale l'idea che possa esserci un rapporto prevalentemente sociale dietro un qualsiasi investimento o dialogo fra le parti in ambito prettamente economico- finanziario. Ed è su questo scambio in rating il punto cruciale su cui faremo affidamento per tutta la durata di questo progetto. Ogni mercato si basa appunto sulla fiducia nei comportamenti dei partecipanti che lo compongono. Per quanto un mercato possa essere regolamentato e controllato, se alla base di esso non c'è il rispetto di un requisito minimo di correttezza, il mercato è destinato a fallire, e con esso anche il numero dei soggetti detentori di un reddito minimo. Analoghe attese di correttezza e rispetto delle regole hanno banche e imprese. L' obiettivo delle nuove regole è aumentare la sensibilità di rischio e la capacità di misurarlo attraverso l'introduzione dei sistemi di rating, consistente in un giudizio sintetico sul merito creditizio di un soggetto debitore e nella conseguente capacità di questo di rimborsare nei tempi prestabiliti capitale e interessi. II rating diventerà una variabile strategica per regolare l'accesso al credito, le imprese con un buon rating vedranno migliorare la loro condizione di accesso al credito, mentre le aziende più rischiose subiranno una restrizione del credito ed un aumento dei relativi tassi d'interesse applicati. Possiamo affermare, quindi, che rientrano nelle prospettive di questa ricerca i rapporti sociali che si instituiscono nelle trattative banche-imprese e quanto questi stessi siano influenti nel successo finanziario di entrambi all'interno dello scenario economico in cui si trovano ad agire in relazione al sistema finanziario vigente, cercando di non sottovalutare il tasso di attività, ossia il rapporto tra i soggetti appartenenti alle forze di lavoro e la popolazione in età lavorativa, ed il tasso di occupazione, ossia il rapporto tra gli occupati e la popolazione in età lavorativa, i quali sono necessariamente direttamente proporzionali a quel sopradetto successo finanziario. La risoluzione di problematiche che possono sorgere durante la stipula di un rapporto economico sono il punto su cui incentrare l'attenzione dei ricercatori al fine di rendere più efficace la regolamentazione vigente sulla vigilanza bancaria. 


\subsection{Comitato di Basilea: sintesi di esigenze diverse}

II Comitato di Basilea per la vigilanza bancaria è un organismo di consultazione che si riunisce periodicamente presso la Banca dei Regolamenti Internazionali con sede, appunto, a Basilea presso la B.R.I. Esso fu istituito nel 1974 dalle banche centrali dei paesi appartenenti al G10, a seguito di un avvenimento rimasto a lungo nella memoria del mercato. II fallimento della tedesca Bankhaus Herstatt (METELLI 2005, p.1-2). La gravità di quel fallimento condiziona sin dal principio i lavori del Comitato, deputato ad intervenire per supportare il buon funzionamento e la stabilità del sistema finanziario globale. II comitato è formato dai rappresentanti delle autorità responsabili della regolamentazione per la vigilanza sul business bancario ${ }^{1}$. Esso non ha potere legislativo e/ o normativo, ma formula proposte e linee guida orientate a due fondamentali obiettivi:

a) Estendere la regolamentazione di vigilanza a tutte le istituzioni bancarie nel maggior numero possibile di paesi;

b) Rendere più efficace la regolamentazione di vigilanza bancaria al fine di assicurare stabilità al sistema complessivo.

Nel 1988 il Comitato raggiunse un primo accordo sui requisiti patrimoniali minimi delle banche con lo scopo di limitare la condotta troppo aggressiva di alcuni istituti di credito, liberi di agire in contesti normativi poco regolamentati. Nacque così Basilea1. Per prevenire, circoscrivere e vigilare sui fenomeni della mancanza di fiducia nei soggetti coinvolti nei mercati finanziari, il comitato di Basilea1 definii i rischi che le banche possono incontrare e stabilì i contenuti che le banche stesse dovevano seguire per tutelarsi da tali rischi. La nozione di rischio evoca "l'eventualità di un andamento sfavorevole nello svolgimento di un'azione futura, contrario rispetto al conseguimento delle finalità dei soggetti preposti al governo dell'azienda stessa. La normativa sull (SASSI 1940, p.2)'adeguatezza delle banche si fonda sui seguenti semplici principi:

\footnotetext{
${ }^{1}$ I membri del comitato provengono dai seguenti paesi: Belgio, Canada, Francia, Germania, Giappone, Italia, Lussemburgo, Paesi Bassi, Regno Unito, Stati Uniti, Spagna, Svezia, Svizzera.
} 
a) Ogni attività posta in essere dall'impresa bancaria comporta l'assunzione di un certo grado di rischio

b) II rischio deve essere quantificato e supportato da capitale (che deve essere "adeguato")

c) Con il termine "adeguatezza patrimoniale" si intende che, il patrimonio debba essere adeguato ai rischi assunti. L'accordo di Basilea1 mirava a costituire una dotazione patrimoniale adeguata a fronteggiare principalmente il rischio di credito connesso con le esposizioni in e fuori bilancio (ONADO 2004, p.64-65). II coefficiente minimo dell'8\% fra patrimonio di vigilanza e attività ponderate in base al rischio, veniva ritenuto sufficiente ad offrire una copertura implicita nei confronti di altri rischi bancari: di mercato, operativo, di liquidità, legale e di reputazione. I cosiddetti "coefficienti patrimoniali minimi obbligatori". Ai fini del patrimonio di vigilanza Basilea1 prevedeva due tappe obbligatorie, ossia ponderare l'attivo per il rischio di credito e calcolare il patrimonio di vigilanza. Per patrimonio di vigilanza si intende il capitale idoneo alla copertura dei rischi ed è composto dal patrimonio di base più il patrimonio supplementare ${ }^{2}$.

\subsection{I limiti di Basilea1}

I limiti di Basilea1 li possiamo riassumere in 5 punti fondamentali:

a) Le misure di rischio sono poco differenziate. La rudimentalità del vigente sistema di ponderazioni non consente di istituire una stretta correlazione fra il rischio d'insolvenza specifico di una determinata controparte e la relativa copertura patrimoniale.

b) Non si riconosce il ruolo svolto dalla diversificazione di portafoglio quale fattore correttivo del rischio. La diversificazione è in grado di ridurre il rischio complessivo del portafoglio, e ci ha un impatto particolarmente

\footnotetext{
${ }^{2}$ II patrimonio di base è costituito dal capitale versato, dalle riserve, dal fondo per rischi bancari. (Unicredit 2005)
} 
significativo sulle banche che hanno impieghi ben diversificati, quali le banche specializzate nel mercato retail e nelle Piccole e medie imprese.

c) Le misure di rischio sono statiche. Le tabelle di coefficienti sono poco differenziate ed invariabili.

d) II rischio viene considerato insensibile alla struttura per scadenze e quindi non viene pesato il fatto che un'esposizione creditizia presenti un grado di rischio diverso a seconda della vita residua.

e) Infine, rimangono escluse le altre tipologie di rischio, che sono comunque presenti nell'attività bancaria (METELLI 2005, p.17-18).

\section{L'evoluzione degli accordi di Basilea}

Dai limiti di Basiela1 il comitato decise di istituire una successiva normativa, attuando dei fondamentali cambiamenti e rinnovando alcuni parametri che erano alla base del precedente accordo. Tutto questo venne definito Basilea2. II Comitato di Basilea2 è un organismo propositivo che non ha il potere di emanare norme cogenti. Ciò nonostante, l'autorevolezza riconosciuta al Comitato fa sì che le sue proposte siano attentamente vagliate dai soggetti competenti. Tra tali soggetti figurano I'Unione Europea e i suoi organismi, la cui Commissione Europea ha partecipato sin dall'inizio ai lavori di Basilea in qualità di osservatore. Al consiglio Europeo spetterà emanare una direttiva, definendo le date attese affinché essa sia recepita nella legislazione dei singoli paesi dell'Unione Europea (METELLI 2005, p.24-25). I prodromi della creazione del Comitato di Basilea2, vengono fatti risalire al giugno 1998, quando il Consiglio Europeo di Cardiff ha chiesto alla Commissione Europea di presentare, prima del successivo incontro a Vienna, un quadro di azione teso a migliorare il mercato unico dei servizi finanziari. La Commissione risponde nell'ottobre del 1998 con l'elaborazione di un quadro di azione, individuando una serie di temi urgenti da esaminare, al fine di ottimizzare il funzionamento del mercato finanziario europeo e beneficiare dei vantaggi connessi all'introduzione della moneta unica europea. Queste attività preparatorie introducono al Financial Services Action Plan, che mira a realizzare mercati integrati per i servizi finanziari nella UE. I primi tre anni di gestazione (2000-

Revista do Direito UNISC, ISSN: 1982-9957

Santa Cruz do Sul №. 47 | p. 93- 124 | SET-DEZ 2015

https://online.unisc.br/seer/index.php/direito/index 
2003) sono stati scanditi dal rilascio di successive versioni, costantemente aggiornate, della proposta originaria, sia da parte dello stesso Comitato di Basilea, sia da parte della Commissione Europea. Nel 2003 ha assunto notevole importanza il QIS3 (Quantitative Impact Study), uno studio che le principali banche di tutti i Paesi hanno svolto, sotto il controllo delle rispettive vigilanze, per misurare con la massima precisione possibile gli effetti attesi dal nuovo impianto normativo. I risultati sono stati utilizzati per la "calibrazione" finale dell'accordo. La Commissione Europea, contestualmente alla pubblicazione del suo terzo documento di consultazione, presentato a luglio 2003, (Based Commettee's Third Consultative Paper), ha richiesto commenti e osservazioni pubblici alla comunità finanziaria e bancaria europea. Le osservazioni pervenute alla Commissione, sono servite poi, a modificare le regole in corso di revisione, e tengono conto delle specificità dell'Unione Europea per essere recepite nella nuova direttiva europea sulla capital adequancy. Solo dopo aver raccolto ed analizzato tutti i pareri della comunità finanziaria europea, la Commissione ha completato la stesura definitiva della direttiva (che era attesa nel 2005). II 29 novembre del 2005, l'Europarlamento ha adottato la nuova direttiva sui requisiti di capitale degli istituti di credito che recepisce sostanzialmente i termini dell'accordo raggiunto al G10 su Basilea2. $L$ 'iter di approvazione è terminato con il giudizio finale dell'Ecofin di novembre. Per quanto concerne l'ambito di applicazione della norma, l'intendimento iniziale del Comitato di Basilea era quello di circoscriverne l'attuazione alle grandi banche internazionali. II successivo dibattito, guidato in particolare dalla Commissione Europea, è invece pervenuto ad un altro indirizzo, suggerendo che il provvedimento riguardi l'intero sistema bancario. Gli Stati Uniti hanno dichiarato, in diverse occasioni nel corso del 2003, che intendono muoversi nel solco della proposta originaria ed a complicare il quadro è giunta la dichiarazione di Cina ed India che non intendono aderire all'accordo. Si tratta di un processo che, pur incontrando parecchi ostacoli, comunque proseguirà. Esiste un problema relativo ai tempi di attuazione su scala sufficientemente ampia; in particolare, rimane qualche incertezza circa l'entrata in vigore della nuova normativa nei paesi diversi da quelli appartenenti al G10. Per facilitare la pianificazione del passaggio a Basilea2, il Comitato ha chiesto ad un gruppo di autorità per la vigilanza, al Fondo Monetario Internazionale ed alla Banca 
Mondiale, di elaborare un programma di assistenza ai Paesi esterni al G10. A tale scopo, il Comitato ha istituito l'Accord Implementation Group per facilitare lo scambio di informazioni tra organismi di regolamentazione, e la Capital Task Force, gruppo di lavoro cui spetta di ponderare modifiche e interpretazioni significative del nuovo accordo (METELLI 2005, p.26-29).

\subsection{L'accordo di Basilea2: i nuovi fondamenti}

Basilea2 con il suo rielaborato impianto regolamentare si prefigge di porre rimedio alle carenze lamentate facendo perno sui seguenti parametri:

a) Mantenere un livello complessivo di patrimonializzazione del sistema almeno pari a quello attuale.

b) Favorire la parità concorrenziale a livello internazionale e migliorare la sensibilità al rischio, definendo requisiti patrimoniali fondati su una misurazione più accurata e completa dei rischi.

c) Definire principi valevoli per tutte le banche, al fine di promuovere la stabilità del sistema attraverso l'adozione di regole di vigilanza uniformi.

d) Abbandonare un approccio rigido e indifferenziato all'adeguatezza patrimoniale. II nuovo accordo consta di tre parti (denominate pilastri) che si rafforzano reciprocamente. Essi sono:

1) II rischio operativo;

2) II controllo delle banche centrali;

3) La disciplina del mercato e la trasparenza.

In definitiva, premesso che come sopra detto è previsto un lungo periodo transitorio di adeguamento, la mission del futuro accordo di Basilea3 è semplice: tutte le operazioni che una banca compie (vendita di titoli, erogazione di crediti ecc.) comportano dei rischi, e quindi delle possibili perdite. Più forte è il rischio, più alte potrebbero essere le perdite, e maggiore è la quantità di denaro che la banca deve accantonare per tutelarsi, e che non può utilizzare in nessun modo: sono soldi in cassaforte da tenere per le emergenze. Le nuove disposizioni, che cadono in un contesto ancora incerto per l'economia, aumentano in percentuale il capitale che gli istituti di credito devono accantonare per "sicurezza". Le banche dovranno quindi sostenere un incremento dei costi e conseguentemente questo finirà per ricadere sui loro Revista do Direito UNISC, ISSN: 1982-9957 
clienti in termini di aumento delle commissioni e degli spread sui prestiti bancari. Alla politica comunitaria spetta l'arduo compito di concepire delle strategie economico-sociali che mettano in risalto la diversità degli stati membri, considerandone virtù da salvaguardare in un quadro unico che anche dal punto di vista politico-costituzionale di fatto ancora non esiste.

\section{I metodi}

La metodologia d'analisi utilizzata in questo studio consta l'aspetto quantitativo e qualitativo dell'accesso al credito in un panorama inequivocabilmente nuovo $e$ in continuo mutamento dell'economia internazionale. Tecnicamente ci siamo soffermati sull'utilizzo del "rating", strumento coniato dal comitato di Basilea che misura lo stato di salute economica di ogni qualsivoglia azienda, da tempo largamente diffuso. Lo studio che in prima istanza riguarda il rapporto tra banche e imprese, con le loro asimmetrie informative, si riflette sulle problematiche sociali degli attori coinvolti nel mercato economico tutto. Un'informazione corretta nelle attività economiche, in base ai principi di trasparenza, efficienza ed economicità, fa sì che le risorse "finite" del nostro globo vengano sfruttate nel miglior modo possibile. Valorizzare le diversità dei popoli e le esigenze degli individui salvaguardando e rispettando ogni essere che abita questa terra è una prerogativa verso cui tutti dobbiamo tendere. In definitiva uno sviluppo sostenibile tout court. Nel paragrafo precedente abbiamo citato i tre pilastri su cui si fonda Basilea2 ossia: il rischio operativo, II controllo delle banche centrali, la disciplina del mercato e la trasparenza. Questi tre pilastri rappresentano un criterio metodologico su cui fondare lo studio di un'analisi scientifica di mercato.

\subsection{II rischio operativo}

"Il Rischio Operativo è definibile come il rischio di perdite derivanti dall'inadeguatezza o dalla disfunzione di procedure, risorse umane e sistemi interni, oppure da eventi esogeni. Tale definizione include il rischio legale che comprende, tra l'altro, l'esposizione ad ammende, sanzioni pecuniarie o penalizzazioni derivanti da provvedimenti assunti dall'organo di vigilanza, ovvero da regolamenti privati" (COMITATO DI BASILEA 2006, PAR. V). Per il

Revista do Direito UNISC, ISSN: 1982-9957

Santa Cruz do Sul №. 47 | p. 93- 124 | SET-DEZ 2015

https://online.unisc.br/seer/index.php/direito/index 
rischio operativo il Comitato propone un trattamento articolato in tre metodologie che riguardano standard qualitativi e quantitativi (BANCA DEI REGOLAMENTI INTERNAZIONALI 2003, p.120). In ordine crescente di complessità e sensibilità al rischio esse sono:

A. Metodo dell'indicatore semplice (basic indicator approach)

B. Metodo standard (standard approach).

C. Metodo avanzato (advanced measurement approach - AMA).

A. II primo approccio "basic indicator approach" richiede alle banche di detenere, a fronte del rischio operativo, un ammontare di capitale pari al $15 \%$ del margine d'intermediazione medio dell'ultimo triennio. La ratio è che banche più grandi, con un "fatturato" più elevato, sono proporzionalmente soggette a maggiori rischi. Questa metodologia è di rapida applicazione, ma molto criticabile. Da un lato, non tiene conto del fatto che alcune aree di attività della banca sono più vulnerabili di altre al rischio operativo (per esempio, la negoziazione titoli comporta elevati margini d'incertezza connessi a errori umani, a frodi e al regolare funzionamento delle procedure elettroniche di regolamento, mentre la raccolta di depositi a risparmio presenta rischi assai più contenuti); dall'altro, non "premia" in nessun modo le banche che investono in procedure di controllo del rischio operativo più sofisticate.

B. Per tenere conto della prima critica, Basilea2 introduce il secondo approccio, detto "standard approach". Esso prevede che il margine d'intermediazione della banca venga sezionato in otto linee operative:

- corporate finance,

- Trading \& sales (negoziazioni e vendite di strumenti finanziari),

- servizi di pagamento,

- commercial banking,

- gestioni fiduciarie,

- retail banking,

- asset management,

- negoziazione di titoli al dettaglio. 
C. Per le prime tre, visto il loro maggior contenuto di rischio, il coefficiente del $15 \%$ viene alzato al $18 \%$; simmetricamente, il coefficiente scende al $12 \%$ per le ultime tre, ritenute meno rischiose. II comitato di Basilea ha deciso di autorizzare anche un terzo approccio, chiamato "metodo avanzato". I parametri e criteri di calcolo del capitale dell'approccio avanzato non vengono indicati espressamente: ci si limita invece a prevedere una serie di requisiti minimi, che dovranno essere soddisfatti dai sistemi "avanzati" realizzati dalle singole banche (RESTI 2003, p.2324). Ad esempio, il sistema dovrà essere in grado di coprire un elenco di tipologie di perdite operative espressamente indicato dal nuovo accordo, dovrà misurare $i$ singoli fattori di rischio in modo sufficientemente capillare, dovrà determinare il patrimonio in misura tale da coprire perdite attese e inattese, dovrà basarsi su dati storici e processi di analisi credibili, trasparenti, ben documentati e verificabili. La scelta di non specificare uno schema di misurazione preciso, ma solo dei requisiti minimi, risponde a due finalità: da un lato, si prende atto del fatto che le tecniche di stima del rischio operativo sono ancora molto giovani, e si vuole evitare di "congelarle" in una fotografia che potrebbe risultare superata, nei prossimi anni, da prassi operative più evolute; dall'altro, si vuole incentivare le banche ad investire in queste tecniche senza vincolarle ad una particolare soluzione tecnica (RESTI 2003, p.23-24).

Per rischio operativo intendiamo il rischio di subire perdite derivanti dall'inadeguatezza o dalla disfunzione di procedure, risorse umane e sistemi, oppure da eventi esogeni. Tale definizione ricomprende anche il rischio legale. Sono invece esclusi i rischi strategici e di reputazione. Nei lavori iniziali del Comitato di Basilea, la definizione dei rischi operativi era, per così dire, "in negativo": si considerava rischio operativo tutto ciò che non rientrava nelle categorie meglio note e classificate di rischio di credito e di mercato. II Comitato di Basilea, infine, è riuscito a elaborare una nozione "in positivo", definendo il rischio operativo come "il rischio di perdite conseguenti a inadeguati processi interni, errori umani, carenze nei sistemi operativi o a causa di eventi esterni" (LOCATELLI, MAGISTRETTI, SCALERANDI 2001, p.4-5). Per il momento, ci limiteremo a dire che data la novità dell'introduzione del rischio operativo, in realtà nessuno ha cognizione di quanto esso inciderà sui requisiti patrimoniali 
richiesti al sistema bancario. Possiamo solo dire che il suo avvento sarà tanto meno traumatico ed oneroso quanto più i sistemi di rating, e soprattutto i sistemi di rating interni che dovranno adottare le banche, sapranno portare un'effettiva riduzione del requisito del rischio creditizio.

\subsection{II controllo delle banche centrali}

In Basilea2 il capitale continua a rappresentare, come nell'accordo precedente, la principale forma di presidio dei rischi. Tuttavia, il nuovo accordo osserva che la copertura dei rischi posta in essere da una banca risulta tanto più efficace quanto più solidi e completi sono i suoi processi interni di controllo, i limiti operativi introdotti a guardia dell'operatività di singoli uffici o divisioni, le politiche di accantonamento a riserva e di pianificazione dei futuri fabbisogni patrimoniali. E' dunque previsto che le Autorità nazionali di vigilanza svolgano un'azione di controllo prudenziale presso le singole banche, per incoraggiarle nell'elaborazione e nell'uso di tecniche di misura e gestione del rischio migliori (RESTI 2003, p.24-25). Questa azione rappresenta il "secondo pilastro" di Basilea2. II controllo prudenziale deve ispirarsi a quattro principi d'azione fondamentali:

a) Verificare che ogni banca disponga di un procedimento per determinare la propria adeguatezza patrimoniale complessiva, in rapporto al suo profilo di rischio, e di una strategia per il mantenimento di adeguati livelli patrimoniali;

b) Riesaminare e valutare tale procedimento, adottando appropriate misure prudenziali qualora esso non risulti pienamente soddisfacente;

c) Imporre alla banca requisiti patrimoniali ulteriori, ove necessario, rispetto a quelli minimi quantificati nel primo pilastro,

d) Svolgere il proprio intervento in maniera tempestiva e precoce, evitando che il patrimonio della banca scenda al disotto dei livelli compatibili con il suo profilo di rischio ed esigendo l'adozione di pronte misure correttive se la dotazione di patrimonio non e mantenuta o non viene ripristinata (BANCA DEI REGOLAMENTI INTERNAZIONALI 2003, p.138-153).

\subsection{La disciplina del mercato e la trasparenza}


Il terzo pilastro, che riguarda la disciplina di mercato, mira a fornire al mercato tutte le informazioni necessarie per sanzionare le banche che dispongano di una dotazione patrimoniale insufficiente o di un complesso di procedure inadeguate a fronteggiare i rischi posti in essere. A tal fine, obbliga le banche a rendere pubbliche alcune informazioni fondamentali di carattere quantitativo e qualitativo. Per evitare di caricare le banche di eccessivi compiti, e di inondare il mercato con un flusso di notizie poco significative, Basilea2 precisa che l'obbligo di trasparenza riguarda solo le cosiddette informazioni rilevanti (tali, cioè, che la loro omissione o errata indicazione potrebbe modificare o influenzare il giudizio o le decisioni di chi su di esse fa affidamento) e fornisce un elenco dei principali dati da rendere pubblici. Le segnalazioni previste dal terzo pilastro hanno di norma cadenza semestrale (annuale per le informazioni qualitative su politiche, obiettivi, sistemi di reporting e gestione della banca; trimestrale per i coefficienti patrimoniali delle grandi banche e i principali aggregati del calcolo). Vengono invece escluse dall'obbligo di trasparenza le informazioni esclusive e confidenziali che, se rese note alla concorrenza, possono diminuire il valore della banca intaccando in tal modo la sua posizione competitiva; si tratta comunque di esenzioni limitate e motivate (BANCA DEI REGOLAMENTI INTERNAZIONALI 2003, p.154-170). Concludendo la descrizione dettagliata dei pilastri che compongono Basilea2 passiamo adesso ad analizzare un altro fattore di cruciale importanza, il rating.

\section{Il sistema di rating}

La parola Rating, che significa letteralmente "valutazione" (dall'inglese to rate), è un indice che sintetizza, attraverso una sigla convenzionale alfabetica o alfanumerica, la capacità di un certo soggetto di far fronte ai propri debiti nei successivi 12 mesi. Quest'ultimo è, quindi, un indicatore puntuale, cioè riferito ad un preciso momento, e non stazionario, infatti è in continua evoluzione e, per tale motivo, deve essere monitorato periodicamente (www.eurating.it). In base a quanto stabilito da Basilea2 le banche dovranno accantonare quote di capitale proporzionali al rischio derivante dai vari rapporti di credito assunti, valutati attraverso lo strumento del rating. $A$ maggior rischio dovrà 
corrispondere maggior accantonamento e, di conseguenza, maggiori costi. La necessità delle banche di dover valutare il rischio di concessione del credito in funzione del rating di ogni singolo richiedente è data dal fatto che la percentuale che le banche eroganti dovranno accantonare sarà più alta in funzione di rating più bassi e viceversa; ne consegue che le aziende richiedenti dotate di un rating basso avranno sempre più difficoltà nell'accesso al credito e comunque dovranno pagare un prezzo più alto in termini di oneri finanziari (www.basileadue.it). II rating condizionerà quindi la concessione del credito e $\mathrm{i}$ relativi costi e permette correttezza, trasparenza e fiducia nel rapporto clientebanca. Sappiamo che con Basilea1 i coefficienti di ponderazione variavano solamente in base all'identificazione della natura della controparte debitrice, il rischio paese e le eventuali garanzie ricevute a fronte del debito. Essi erano applicati in maniera meccanica, in base ai fattori statici, prescindendo dalla reale natura della controparte debitrice. II nuovo requisito patrimoniale punta a discriminare meglio tra debitori di diversa qualità, conducendo a risultati molto più bassi o molto più alti del vecchio $8 \%$ a seconda del rating, cioè del voto assegnato al grado di affidabilità del prenditore. Sorge dunque un primo problema: a chi spetta il compito di assegnare un simile rating? L'accordo di Basilea2 prevede due possibili soluzioni: Le banche più piccole potranno fare riferimento a rating esterni, assegnati da agenzie specializzate come Moody's, Standard \& Poor's o altri soggetti selezionati dalle Autorità di vigilanza; questa soluzione è detta "approccio standard". Le banche maggiori potranno invece costruire i propri rating interni, nel rispetto di regole organizzative e metodologiche rigorose, certificate dalle Autorità di vigilanza. Questo approccio dei rating interni si compone, in realtà, di due diverse metodologie, in ordine crescente di sofisticazione: ad un "approccio di base", pensato per le banche che hanno una limitata esperienza nel rating, si contrappone infatti l'approccio avanzato", riservato a chi, nel tempo, saprà dimostrare alle Autorità di aver sviluppato strumenti di controllo del credito raffinati ed affidabili (RESTI 2003, p.6).

\section{I quattro profili del rischio di credito}

- La probabilità d'insolvenza (probability of default -PD)

Revista do Direito UNISC, ISSN: 1982-9957

Santa Cruz do Sul №. 47 | p. 93- 124 | SET-DEZ 2015

https://online.unisc.br/seer/index.php/direito/index 
Con riguardo alla stima della PD, Basilea2 non entra nel merito di come debba essere costruito il sistema di rating di una banca. Non specifica quindi quali indicatori (per esempio quali indici di bilancio) debbano essere utilizzati nell'assegnazione dei rating, e tanto meno obbliga le banche ad adottare sistemi automatici, basati su tecniche statistiche di scoring; $\mathrm{ci}$ che chiede, piuttosto, è che i criteri di costruzione del rating, così come le basi di dati utilizzate, siano documentati e archiviati in modo trasparente, utilizzati con piena cognizione di causa e rivisti periodicamente in base alla loro efficacia passata. Alle diverse classi della scala di rating interna dovranno essere associati valori crescenti di PD in base all'esperienza passata della banca (cioè alle frequenze di default empiricamente osservate, per le diverse classi, negli anni precedenti). In questo lavoro di "collegamento" tra classi di rating e valori di PD, le banche potranno aiutarsi anche con i risultati di modelli statistici (se presenti), o con le indicazioni (quando disponibili) provenienti da agenzie di rating esterne (BANCA DEI REGOLAMENTI INTERNAZIONALI 2003, p.54).

- La perdita in caso d'insolvenza (loss given default, LGD)

La misura della LGD seguirà criteri diversi a seconda che la banca abbia adottato l'approccio di base o avanzato. Nel primo caso, sarà necessario fare riferimento ad una griglia di valori prefissati, che varieranno in funzione delle garanzie reali associate ai singoli prestiti. Se deciderà di adottare l'approccio avanzato ai rating interni, la banca sarà libera di costruirsi un proprio sistema di misura della loss given default, articolato secondo una scala di più gradini, cui corrisponderanno valori di LGD crescenti. Le LGD associate alle diverse classi della scala dovranno essere stimate facendo riferimento all'esperienza storica della banca, utilizzando valori medi di lungo periodo e tenendo conto del fatto che le LGD potrebbero essere più elevate della media proprio quando l'economia è in recessione, e i default si fanno più frequenti (BANCA DEI REGOLAMENTI INTERNAZIONALI 2003, p.56). In generale, le banche che adotteranno l'approccio avanzato saranno libere di indicare qualunque valore di LGD per i diversi prestiti, purché siano in grado di dimostrarne, dati alla mano, l'attendibilità.

- Esposizione in caso di inadempienza (exposure at default, EAD) 
Quantifica l'ammontare del finanziamento a rischio di inadempienza. EAD equivale a dire: su quale esposizione effettiva si avrà la perdita? La determinazione di questo valore dipende dall'ammontare di fido concesso.

- La durata (maturity, M)

Consiste nella scadenza contrattuale del finanziamento. $\mathrm{Pu}$ rappresentare un fattore critico per la banca nel momento in cui vi sia un non livellamento tra le scadenze dei crediti e i debiti della banca. Anche nell'approccio di base, le Autorità nazionali potrebbero autorizzare le banche all'uso di stime interne della maturity, almeno per alcune categorie di crediti (RESTI 2003, p.12-15). Infine, l'approccio IRB si suddivide alternativamente in:

a) IRB Foundation che è l'approccio di base fondato sui rating interni. Esso prevede che la Banca valuti internamente la probabilità di insolvenza di ciascun prenditore, mentre l'Autorità di Vigilanza fornirà tutti gli altri input del modello di valutazione. In altre parole, la banca deve ottenere preliminarmente la validazione, da parte della Banca d'Italia, del sistema di valutazione interno delle controparti.

b) IRB Advanced con il quale, a differenza del precedente, metterà a disposizione più autonomia alle banche che si dimostreranno in grado di costruire sistemi di rating interni efficienti ed efficaci, consentendo loro di stimare internamente anche i valori di LGD, di EAD.

E' evidente che i metodi adottabili si basano su informazioni risksensitive in base alle quali ponderare oggettivamente le proprie scelte di gestione e di costo delle attività di credito. In buona sostanza, la valutazione della rischiosità dell'impresa si ricava dalla ponderazione dei seguenti elementi:

- strategie ed organizzazione aziendale

- qualità del management

- qualità professionale delle risorse umane

- struttura economico-finanziaria dell'impresa

- qualità dei bilanci e delle altre informazioni gestionali

- completezza e coerenza dei dati gestionali

- dinamica finanziaria a breve e medio-lungo termine

- dinamica della capacità di servizio del debito

A ciò si aggiungono le informazioni sulla condotta dell'impresa nei rapporti con gli istituti di credito (informazioni andamentali). Si valutano, 
pertanto, i debordi in c/c, gli insoluti, i ritardi nei pagamenti delle rate, il numero di c/c attivati, la quantità monetaria gestita sul c/c, ecc.).

Della necessità di migliorare la quantità e la qualità del flusso di informazioni destinate agli intermediari finanziari ci si è resi conto solo dopo gli illustri crack finanziari che hanno coinvolto i principali gruppi industriali italiani, oltre alle principali banche nazionali. Su iniziativa dell'ABI, il sistema bancario ha messo a punto, nell'ambito del progetto "PattiChiari: le banche cambiano", un documento nel quale sono sintetizzate le principali informazioni aziendali richieste nell'istruttoria di affidamento bancario. Si tratta, in sostanza, di descrivere:

- l'impresa ed il suo business

- le finalità del finanziamento

- la capacità di rimborso dell'impresa

- il capitale investito dall'imprenditore o dai soci

- le garanzie per la mitigazione del rischio

- il sistema delle relazioni tra la banca e l'impresa.

Come è evidente, si tratta di informazioni qualitative e quantitative che offrono alla banca una base informativa sufficiente sulla quale esprimere un giudizio di qualità del merito creditizio del cliente (http://www.miranet.it/dossier/strumenti_finanziamento_alternativi_basilea2.html ).

\section{II rating interno}

Concludiamo questa full immersion in Basilea2, prima di passare ai successivi accordi, con il "sistema di rating interno". Per sistema di Rating interno si intende l'insieme di metodi, procedimenti, dati e sistemi informativi che fungono da supporto alla valutazione del rischio di credito, all'attribuzione dei gradi interni di merito, e alla stima quantitativa dell'inadempienze e delle perdite" (BANCA DEI REGOLAMENTI INTERNAZIONALI 2003, p.72). L'aggettivo "interno" si riferisce al fatto che il criterio, anche se mutuato 
dall'agenzia di rating esterna, fa parte di un sistema complessivo sviluppato e gestito all'interno della banca. La novità è duplice:

1) L'introduzione di una scala di merito graduata, il che porta ad un'evoluzione delle tecniche di monitoraggio del portafoglio crediti di una banca.

2) L'introduzione di giudizi standardizzati, facilita il dialogo tra i soggetti coinvolti e una crescente misura di trasparenza da parte della banca (http://agevolazionifinanziarie.consrag.it/forum/rating-prenditore.asp).

\subsection{DIMENSIONI DI ANALISI DEL RATING INTERNO}

Riveste una certa importanza la possibilità di poter ricorrere a sistemi di rating diversi per differenti settori di clientela, lo stesso comitato dispone che il sistema di rating interno debba avere due dimensioni di analisi distinte e separate:

1) L'analisi del rischio d'inadempienza del debitore, riferita alla capacità del cliente di essere solvibile.

2) L'esame delle caratteristiche specifiche dell'operazione di credito, al fine di tenere conto delle peculiarità contrattuali (METELLI 2005, p.74-77). Poiché i requisiti del rating interno sono di tipo qualitativo, le autorità di vigilanza nazionali dovranno valutarne l'osservanza per definire quali banche potranno adottare il nuovo approccio (BANCA DEI REGOLAMENTI INTERNAZIONALI 2003, p.73-75).

Dalle considerazioni svolte finora, si può affermare che Basilea2, attraverso tecniche più rigorose di calcolo del rischio di credito, non è altro che il tentativo di contrastare i possibili fallimenti del mercato insiti nell'attività bancaria (MOSCATO 2005), propria di tutti i processi economici europei e non.

\section{Conclusioni}

In risposta alla recente crisi finanziaria Basilea3 si propone di accentuare un insieme di regole relative prevalentemente alla vigilanza bancaria. II 12 settembre 2010 i Governatori e i Capi delle Autorità di vigilanza del G20 hanno 
approvato le proposte del Comitato di Basilea, che sono state sottoposte ai Capi di Stato e di Governo nel mese di novembre 2010 a Seul. Le nuove regole, che sono state oggetto di un'ampia consultazione con l'industria bancaria, entreranno in vigore all'inizio del 2013. È stato tuttavia previsto un lungo periodo transitorio fino al 1.1.2019 così da favorire un graduale adeguamento delle strategie operative delle banche ed evitare ricadute sulla ripresa economica. L'obiettivo che si persegue con questa riforma è di prevenire l'eccessiva assunzione di rischi da parte degli operatori, rendere il sistema finanziario più solido, stabilire un terreno di gioco davvero uniforme. Le misure riguarderanno unicamente gli intermediari finanziari.

- Introduzione di standard minimi di liquidità

- La definizione di capitale regolamentare unitamente alla fissazione di più elevati requisiti patrimoniali

- Migliore copertura dei rischi di mercato e di controparte

- Contenimento del livello di leva finanziaria

- Misure anticicliche per ridurre la "prociclicità" delle regole prudenziali

\section{I Risultati}

Per definire i risultati previsti o perlomeno ipotizzati dagli accordi di Basilea bisogna ancora valutare alcune variabili, partendo dall'assunto che questa materia di studio non è ancora del tutto definita ed è un campo di ricerca in evolversi, che quindi non ha ancora stabilito un criterio sommario su cui basarsi. Gli accordi di Basilea3, ad esempio, sono ancora in fase di delineazione e non è stato stabilito quando potranno essere attuate e sostituite ai precedenti. A motivo di quanto detto occorre, in questa parte dell'analisi condotta, aggiungere alle informazioni di bilancio (analisi point-in-time) il valore dell'indagine through-the-cicle. Ciò significa valutare variabili quali:

a) L'andamento previsto della domanda:

- Ampiezza giro d'affari.

- Trend storico giro d'affari.

- Stabilità dell'andamento del giro di affari.

- Prospettive di sviluppo del giro di affari. 
- Propensione alla Ricerca \& Sviluppo (Intesa a verificare non solamente quanto si spende in ricerca e sviluppo ed i risultati conseguiti, ma tesa anche a verificare come cambiano i target dell'azienda sul mercato).

- Diversificazione per prodotti, per linee di business e per clienti.

b) L'evoluzione attesa della concorrenza:

- Potere negoziale della distribuzione.

- Sostituibilità dei prodotti.

- Livello di concentrazione dell'offerta.

c) Prospettive di settore e di mercato e dinamica delle esigenze dei clienti;

d) Stato complessivo dell'economia dei paesi di riferimento dell'azienda;

e) Quadro normativo e regolamentare del settore di appartenenza;

f) Livello di competizione e fattori di successo;

g) Rispondenza dell'offerta aziendale alle esigenze dei clienti;

h) Sistemi di controllo di gestione economica e finanziaria;

i) Indicatori di prestazione utilizzati e livello di soddisfazione dei clienti; Stabilità/Ciclicità settoriale (PISTELLI 2001, p.64-66).

\subsection{L' analisi di benchmarking}

Successivamente si procede alla valutazione della posizione dell'azienda relativamente al settore di appartenenza, sulla base di indici economicofinanziari e della posizione competitiva rispetto ai concorrenti di riferimento. L'analisi di benchmarking può quindi essere suddivisa in due fasi:

1) Comparazione degli indici d'impresa con quelli di settore (turnover, ciclo monetario, leva finanziaria, leva operativa, $\mathrm{ROI}$, ecc.);

2) Posizione competitiva: quota del fatturato di settore.

Le aziende con un numero di clienti crescente e diversificato hanno infatti una maggiore probabilità di rispondere velocemente ed efficientemente ai cambiamenti del contesto regolamentare, tecnologico e ambientale e ai cambiamenti macroeconomici e della domanda. Invece, ciò non avviene per le aziende con indicatori deficitari rispetto ai dati medi di settore e con una quota di mercato ridotta, le quali aziende influiscono negativamente sul tasso di occupazione. 


\section{Nuove frontiere in campo metodologico: la valutazione ed il finanziamento dell'innovazione tecnologica}

La normativa di Basilea2 comprendendo sia una analisi quantitativa sia una analisi qualitativa, non è altro che un metodo sofisticato e completo di analisi aziendale.Volendo analizzare le questioni attinenti la valutazione ed il finanziamento dell'innovazione tecnologica e della $R \& S$, sorgono problemi difficili da affrontare poiché, nonostante il loro ruolo per la crescita economica sia ampiamente riconosciuto, sussistono problemi rilevanti nella determinazione del loro valore (ORIANI 2004, p.21-25). A tal fine, è possibile distinguere quattro aspetti critici:

1) L'individuazione dei risultati intermedi delle attività di innovazione tecnologica, rappresentati primariamente da nuova conoscenza e da altre attività intangibili. II capitale di conoscenza dell'impresa può avere uno spettro molto ampio e riguardare diverse categorie di attività intangibili, quali l'innovazione, il capitale umano e le competenze organizzative. Inoltre, la conoscenza è incorporata in diversi "contenitori", quali persone, procedure e sistemi e spesso non è codificata. Infine, la conoscenza tecnologica è strettamente e indissolubilmente legata ad altre attività intangibili.

2) La misurazione delle attività intangibili e l'inadeguatezza della loro rappresentazione in bilancio e negli altri documenti ufficiali.

3) L'incertezza sui rendimenti attesi dagli investimenti in R\&S. Possiamo distinguere così, tre tipi d'incertezza rilevanti per l'analisi economica degli investimenti in R\&S. II primo è di matrice tecnologica, ed è relativo all'effettiva capacità dei progetti di ricerca di generare nuova conoscenza. Il secondo, è di matrice strategica, ed è collegato alla circostanza che l'impresa che investe in $R \& S$ non è sicura di essere la prima a lanciare un nuovo prodotto. II terzo infine, è connesso all'evoluzione di mercato e all'effettiva presenza di potenziali clienti nel momento in cui il nuovo eventuale prodotto è lanciato.

4) L'indeterminatezza delle relazioni causali che dovrebbero legare gli investimenti in R\&S alla performance economica dell'impresa. Infatti, gli investimenti in R\&S rappresentano una misura di input e non di output dei 
processi d'innovazione. Tali investimenti non hanno spesso risultati direttamente osservabili o prevedibili, producendo effetti trasversali e difficilmente isolabili su diversi prodotti e processi. Per concludere, i risultati finali del processo d'innovazione sono spesso il frutto della coevoluzione tra competenze tecnologiche, ambienti istituzionali e preferenze di mercato che non preesistono l'uno all'altro (ORIANI 2004, p. 26-28).

\subsection{Vincoli al finanziamento dell'innovazione tecnologica e della R\&S}

Detto ciò, vediamo quali vincoli presenta il loro finanziamento. E' possibile distinguere almeno due aspetti critici:

1) L'appropriabilità del valore economico della conoscenza. I problemi nascono dall'idea che l'utilizzo della conoscenza non sia esclusivo e che pertanto processi d'imitazione possano ridurre o annullare il vantaggio dell'innovatore, eliminando gli incentivi economici per le imprese a investire in R\&S. Ne consegue così che i rendimenti sociali degli investimenti in ricerca e sviluppo sono maggiori dei rendimenti privati. Infatti, mentre questi ultimi sono limitati al maggior surplus del produttore, i ritorni sociali possono essere misurati dalla somma del maggiore surplus del produttore, del maggior surplus del consumatore e delle esternalità delle quali beneficiano prodotti e processi in altri settori industriali (NELSON 1959, p.297-306).

2) Le asimmetrie informative in R\&S.

Le asimmetrie informative sono particolarmente rilevanti nel campo della $R \& S$ dove l'inventore ha una migliore conoscenza della natura e della probabilità di successo tecnico e commerciale dell'innovazione rispetto ai soggetti esterni che dovrebbero finanziare l'impresa. II problema si accresce notevolmente se a richiedere il finanziamento sono imprese giovani, in assenza di una storia aziendale (AKERLOF 2001). Anche in questo caso quindi, avremo dei fenomeni di moral hazard e di adverse selection:

a) II moral hazard in $R \& S$ 
I problemi di moral hazard relativi all'attività di R\&S nascono dal tradizionale argomento della teoria dell'agenzia e della separazione tra proprietà e controllo (ENSEN, MECKLING 1976, p.305-360). L'idea fondamentale è che i manager siano più avversi al rischio degli azionisti, i quali, avendo la possibilità di diversificare il rischio attraverso opportune strategie di portafoglio, preferirebbero investimenti con combinazioni rischio/rendimento più elevato. E' possibile, invece, che i manager, che sono legati al destino della singola impresa, preferiscano focalizzarsi sui rendimenti di breve termine a sfavore degli investimenti di lungo periodo.

b) Adverse selection in R\&S

L'obbligo di riportare le spese di R\&S immediatamente nel conto economico dell'anno in cui sono sostenute rende più grave il problema delle asimmetrie informative perché genera attività di R\&S fuori bilancio $e$ un conseguente disallineamento tra investimento e benefici attesi. Tale mancata capitalizzazione genera un fenomeno di adverse selection a causa del gap tra investitori informati e investitori non informati. Infatti, gli investitori comprendono bene che i rendimenti degli investimenti in R\&S si estendono oltre il periodo in cui sono sostenuti, ma essendo questi investimenti immediatamente inscritti in conto economico e non differenziando tali spese rispetto agli altri costi operativi, aumenta la dimensione dell'errore nella stima dell'effetto degli investimenti in $R \& S$ sui profitti dell'impresa. L'adverse selection si riflette in un bid-ask spread ${ }^{3}$ sul prezzo dell'azione più elevato per le imprese con una maggiore intensità di R\&S. Lo spread inoltre, dipende positivamente dall'ammontare di attività di R\&S fuori bilancio. In più, tale regime porta a una stima conservativa del reddito per le imprese con un tasso di crescita degli investimenti in R\&S maggiore della loro redditività (tipicamente imprese nelle fasi iniziali del ciclo di vita) e a una stima aggressiva del reddito per le imprese che si trovano nella situazione opposta (tipicamente imprese mature) (ORIANI 2004, p.130-131).

\footnotetext{
${ }^{3}$ Rappresenta la differenza tra la domanda e l'offerta dei titoli azionari. Esso viene usato per misurare la liquidità del mercato (http://www.sella.it/gbs/trading/formazione/glossario/include/b/29_corpo.jsp).
} 


\section{Venture Capital, private equity, direttiva Mifid}

Le considerazioni discusse divengono particolarmente rilevanti per le imprese più giovani e ad alta tecnologia, che risentono maggiormente dei problemi di razionamento e fruizione del credito. Una delle possibili fonti di equity in questi casi è rappresentata dai finanziamenti di Venture Capital (ORIANI 2004, p. 32-35). Con tale espressione s'intende in senso stretto il finanziamento a titolo di equity concesso ad un'impresa con forti potenzialità di crescita che si trovi nelle fasi iniziali della propria vita e che basi la propria strategia sulla commercializzazione di una tecnologia innovativa, sull'entrata in un nuovo mercato o sullo sfruttamento di nuove possibili applicazioni di tecnologie esistenti (RAMPINI 2000, p.130). La società di venture capital ricopre normalmente il ruolo di general partner, assumendo il compito e la responsabilità di organizzare e di gestire il fondo, individuare gli investitori, reperire i capitali e selezionare le opportunità. Gli investitori partecipano nella veste di limited partner, fornendo i capitali e percependo i rendimenti, ma non essendo coinvolti nelle scelte di gestione del fondo. II venture capital facilita il finanziamento delle "piccole" imprese (PIOL 2004, p.15) (per "piccole" imprese qui non intendiamo imprese di piccole dimensioni, quanto "imprese bambine", che nascono piccole, ma aspirano a diventare grandi) in quanto riduce il problema delle asimmetrie informative attraverso l'acquisizione di una conoscenza dettagliata sul settore e sulle imprese finanziate. Dal punto di vista della domanda di finanziamenti, le imprese di nuova costituzione specializzate nei settori tecnologici non hanno bisogno solo di capitale, ma anche di consulenza e di esperienza gestionale. Dal punto di vista dell'offerta sono necessarie invece, soluzioni e forme tecniche di finanziamento appositamente mirate a causa della specificità di tali imprese caratterizzate da un elevato grado di rischio e di informazione poco chiara, soprattutto nei primi stadi di sviluppo. L'asimmetria informativa, l'assenza di garanzie e gli elevati costi di monitoraggio imputabili ai costi di valutazione dei rischi tecnologici, richiedono l'impiego di capacità e professionalità altamente specializzate difficilmente conciliabili con un tradizionale contratto di debito bancario (GOMPERS 1995, p.1461-1489). Sembra che solo alcuni settori siano in grado di beneficiare degli 
investimenti in venture capital, in quanto sono gli unici in cui il regime di appropriabilità dell'innovazione favorisce le "piccole" imprese. Inoltre i venture capitalists contribuiscono al finanziamento dell'impresa solo nelle sue fasi iniziali, richiedendo che nelle fasi successive le imprese possano tornare sui mercati azionari ad alto contenuto tecnologico per ottenere nuove risorse finanziarie per l'innovazione. L'esistenza di un mercato azionario efficiente favorisce per altro anche l'uscita di parte dei venture capitalists che, attraverso il meccanismo della quotazione nell'indice NASDAQ delle nuove imprese, possono monetizzare l'investimento originariamente effettuato. Pertanto, in un sistema economico lo sviluppo dei mercati azionari è legato a quello degli investimenti in venture capital. Fenomeno per certi aspetti simile è il private equity. (METELLI 2005, p.242-245). Anch'esso mira ad aprire il capitale azionario (equity) a soggetti terzi. Si differenzia rispetto al venture capital perché non è rivolto ad "imprese bambine" ma ad imprese già esistenti in presenza di determinate caratteristiche come:

- Dimensioni oltre una soglia minima ( ad esempio fatturato intorno ai 2025 milioni di euro).

- Posizione di forza competitiva in una nicchia di mercato.

- Buone prospettive di sviluppo.

- Performance soddisfacenti.

- Assenza di criticità connesse a ricambi generazionali, etc... I soggetti oggi attivi nel private equity italiano sono:

- Fondi chiusi di diritto italiano.

- Finanziarie di partecipazione.

- Banche.

- Operatori Pubblici.

- Vari operatori stranieri.

La creazione di valore è l'obiettivo dell'investitore che spesso mira alla quotazione finale per ottenere una plusvalenza. Anche in questo caso, lo sviluppo ed il buon funzionamento dei mercati azionari risulta fondamentale per la buona riuscita di queste operazioni. Un'ultima annotazione: le problematiche insite allo sviluppo e al buon funzionamento dei mercati azionari sono destinati ad avere un rapido sviluppo in seguito all'emanazione della Direttiva 2004/39/Ce meglio conosciuta come Direttiva Mifid (Market in financial 
instruments directive, (2004) che, approvata dal Parlamento europeo il 21 aprile 2004, dovrà essere recepita dagli stati membri entro il 2007 e mira a disciplinare l'attività degli intermediari ed i servizi di investimento. Essa, tra l'altro, prevede che non vi sia più l'obbligo di concentrazione degli scambi azionari sulle borse ufficiali nazionali e l'istituzione di "Sistemi di Scambio Organizzato" ( SSO o MFT nel linguaggio Mifid) (DE CRISTOFANO, 2005). Una sola cosa preme sottolineare: l'adozione della direttiva da parte degli Stati membri potrebbe essere un'ulteriore opportunità per favorire l'ingresso di capitale di rischio per finanziare le imprese e le banche, e con esse incrementare la soglia di occupazione specie in Italia. Essi nell'organizzare questi "Sistemi di Scambio Organizzato", avranno sicuramente un ruolo di primaria importanza.

\section{Conclusioni}

E' necessario in definitiva definire quanto segue: i pregi di questo studio sono quelli di informare in modo qualitativo e quantitativo sull'esistenza dei principi basilari degli Accordi di Basilea. Partendo dai pilastri portanti di questi assunti abbiamo tracciato le linee guida dei processi economici attuali, i quali subiranno continui mutamenti considerando una lunga fase di assestamento in particolar modo nell'adeguamento delle aziende alle nuove direttive comunitarie. Ci si chiede sostanzialmente quali siano i benefici che attraverso i nuovi accordi il mercato internazionale potrà tarme, e soprattutto quanto il tasso di occupazione nazionale in primis verrà influenzato positivamente da questi processi. Le aziende dovranno aspettarsi anche di essere esaminate con più attenzione di prima quando richiederanno denaro a un istituto di credito. Le valutazioni del rating delle imprese saranno sempre più attente e sempre più determinanti rispetto ad almeno due decisioni: se e quanto denaro prestare e a quali costi e condizioni farlo. II risultato atteso peculiare è appunto il seguente: agevolare la ricerca ad uno studio attento delle nuove prerogative, frutto di un'analisi in continua evoluzione nata dall'impulso che lo stesso comitato di Basilea ha voluto evidenziare nell'unione europea. Prima di chiedersi cosa le imprese devono fare per prepararsi a Basilea3 è necessario domandarsi: le imprese sono preparate ad un nuovo modo di concepire l'organizzazione 
dell'impresa in relazione al cambiamento epocale in atto? II mercato economico e l'assetto sociale internazionale è pronto ad accogliere nuovi regolamenti attuali e futuri? Quanto i processi economici in relazione al tasso di disoccupazione verranno influenzati positivamente da queste innovazioni?

Possiamo appunto annoverare fra i limiti del nostro studio proprio l'incapacità di rispondere in maniera univoca a questi quesiti, in quanto è ancora in atto un totale riassetto sociale ed economico dell'ambiente in cui gli attori dei più volte citati processi economici si trovano ad agire.

Cosa devono fare le imprese? Primo fra tutti conoscere le nuove "regole del gioco" che il mercato, adesso, certamente più grande e più aperto, determina considerandone altresì gli aspetti culturali. Da un punto di vista tecnico: cambiare il rapporto con la propria banca e sviluppare maggiore cultura finanziaria, allineandosi a più adeguate modalità di comunicazione finanziaria (report gestionali e finanziari, piani strategici, ecc.), dotandosi di strumenti per il calcolo del proprio rating, sviluppando le attività di pianificazione economicofinanziarie e di simulazione interna, considerando l'aumento della "patrimonializzazione" come un obiettivo fondamentale della propria strategia. L'obiettivo di Basilea3 è creare un sistema economico e finanziario più forte, con banche più solide e queste lo saranno anche nella misura in cui le imprese loro clienti saranno più solide. Stare sul mercato oggi è sicuramente "un'impresa" ma, se si vuole avere un'affidabile garanzia di continuità per il futuro, questo non può essere fatto qualora contemporaneamente non si misura e si contiene il proprio rischio aziendale. E' necessaria non solo, in ultima analisi, una attenta pianificazione, ma una continua attività di simulazione degli scenari futuri a fronte del cambiamento di alcuni parametri fondamentali di riferimento, che abbiamo già ampiamente elencato. Risulta pertanto fondamentale effettuare delle analisi di sensitività (analisi "What if"), ossia verificare come possono cambiare i risultati finali al modificarsi di alcuni parametri di riferimento (fatturato, livello dei costi fissi/ variabili, giorni medi di incasso e pagamento, tassi di interesse ecc.). In questo modo si possono simulare a priori i possibili scenari futuri, effettuando di fatto degli Stress Test, per capire fino a che punto può spingersi la capacità di tenuta dell'azienda in presenza di condizioni avverse. 


\section{Discussione}

Negli ultimi anni gli sviluppi teorici della teoria dell'informazione hanno rinnovato l'interesse verso il rapporto banca-impresa, inteso come meccanismo di superamento dell'asimmetria informativa tra creditore e debitore e, dunque, fattore che influenza le condizioni di offerta del credito bancario. Le banche, non solo quelle italiane, si sono trovate ad operare in un contesto di maggiore dinamismo dei mercati, in seguito al processo di trasformazione dell'economia e della concorrenza internazionale. Oggi le banche devono cercare di essere più competitive conseguendo, da un lato maggiori livelli di efficienza per competere nei mercati dei servizi tradizionali, che sono entrati nella fase di maturità, dall'altro lato lo sviluppo dell'innovazione per competere nei mercati nuovi ed emergenti (BARAVELLI 2003, p.10-15). L'introduzione della moneta unica ha costituito poi, un ulteriore pressione al miglioramento dell'efficienza e all'accelerazione del processo di cambiamento. II rating, che potrebbe presentarsi in questa rivoluzione del credito come una ghigliottina per le imprese, può invece svolgere, da un lato, un compito di ridefinizione dei rispettivi confini nelle relazioni tra banche e imprese, e, dall'altro lato, potrà divenire uno strumento preciso di valutazione delle possibilità di crescita e di diversificazione per il management imprenditoriale. Padroneggiare il concetto di rating, saper differenziare le proprie fonti di finanziamento e possedere una strategia finanziaria adeguata al proprio business diventeranno, sempre più nei prossimi anni, fattori strategici per il successo aziendale. In tutto questo, le banche sono chiamate a rivestire un ruolo "proattivo", nel senso che non dovranno essere più semplici risolutori di problemi posti dal cliente, ma potranno rivestire un ruolo fondamentale nell'accompagnare l'imprenditore, soprattutto le PMI, a delineare una propria strategia finanziaria (PARISI 2005, MOSCATO 2005). Gli accordi di Basilea2 a partire dal 2007 e poi 3 quelli successivi, apporteranno molti elementi innovativi nello scenario del mercato finanziario e nelle relazioni creditizie tra banche e imprese. Esse introdurranno delle nuove tecniche che rivoluzioneranno il comportamento delle banche nella gestione del rischio di credito e avranno un impatto significativo sul rapporto banca-impresa e in modo particolare su quello delle PMI. Anche queste ultime riceveranno un rating a seconda del loro grado di solvibilità, in base al quale 
vedranno modificarsi i criteri di erogazione dei crediti e degli interessi praticati. La nuova proposta degli accordi di Basilea contiene, da un lato, le nuove modalità di valutazione e concessione del credito allo scopo di rendere più trasparente il rapporto intercorrente tra le banche, le imprese e i loro rispettivi investitori e, dall'altro, pone le basi per una revisione del sistema creditizio incentrata verso una sempre maggiore solidità e stabilità del sistema bancario. In pratica, sia le imprese di qualsiasi ordine e dimensione, sia le istituzioni e le banche verranno direttamente coinvolte in un processo di efficienza, fonte di significativi cambiamenti e trasformazioni. II punto saliente del nuovo accordo è proprio la capacità di misurare il rischio di credito al fine di garantire maggiore stabilità al sistema finanziario; si pone, cioè, maggiore attenzione agli aspetti qualitativi piuttosto che a quelli quantitativi come misura del rischio. Basilea2 ha avviato, in questo modo, un processo che ha come conseguenza una profonda evoluzione culturale, sia per il sistema bancario sia per quello delle imprese. Appare infine opportuno sottolineare che la normativa degli accordi di Basilea, se colta correttamente, può rappresentare una grandissima opportunità per il sistema bancario italiano, in quanto porta verso un miglioramento continuo dell'intero sistema finanziario a fronte dello sviluppo dell'economia reale. II rapporto banca/ impresa sarà maggiormente orientato alla trasparenza reciproca, quindi sarà interesse comune fornire e ottenere informazioni per una corretta valutazione del rischio. II rating diverrà variabile strategica per regolare l'accesso al credito e il costo relativo: le imprese con buon rating vedranno migliorare le condizioni di accesso al credito, le aziende più rischiose subiranno restrizioni e pagheranno tassi più elevati. Verrà enfatizzato il ruolo di consulenza delle banche a favore delle imprese; agli imprenditori si richiederanno strategie di impresa coerenti e trasparenti sia sul piano industriale sia sul piano finanziario, parallelamente alle banche si richiederanno maggiore trasparenza nella valutazione del rating e maggiore coinvolgimento in termini di consulenza, sviluppo di servizi e di soluzioni innovative. In questo contesto le imprese dovranno abituarsi a mutamenti di ordine culturale, gli imprenditori saranno chiamati a gestire il proprio business in maniera matura, non facendo più affidamento sui rapporti "particolari", "personali", "confidenziali". Essi dovranno imparare ad approcciarsi alla banca in termini professionali, elevando il tenore e la qualità delle informazioni che si scambiano. La banca a sua volta 
troverà le difficoltà maggiori nel selezionare, gestire, conservare tutta una serie di informazioni nuove e nel controllare i processi che le trattano. Il motivo è semplice: potremmo anche decidere di continuare a finanziare imprese non produttive ed incapaci di creare valore aggiunto, giustificandone il finanziamento con motivazioni di ordine morale, etico, ideologico, politico, nazionalistico, ma molte restano le domande che non trovano risposta: chi pagherà per il valore distrutto? A chi accollare l'onere per i mancati guadagni? Con quale denaro potremmo "socializzarne" i costi?

Nondimeno, non vogliamo essere pessimisti, perché riteniamo che moltissime delle nostre imprese hanno le capacità per affrontare adeguatamente questa nuova sfida; le difficoltà ci sono, ma non dobbiamo dimenticare il buono che c'è. Le imprese, in particolare, dovranno saper fornire dati certi e articolati e trasparenti che attestino l'andamento delle proprie attività, dai quali le banche ricaveranno un rating, in funzione del quale verrà definito l'entità e il costo del credito ad esse erogabile. Le aziende quindi, dovranno disporre di questi indicatori riguardanti il proprio andamento, che potremmo definire sommariamente come controllo di gestione, soprattutto per conoscere effettivamente i propri risultati in tempo reale, apportare i necessari interventi correttivi e, in ultima analisi essere più competitive. L'importanza degli accordi di Basilea ci pare ancora più stringente, se consideriamo che la sfiducia dei potenziali investitori nei confronti del sistema bancario può produrre, e forse ha già prodotto in questi anni, fenomeni pericolosi come l'avvento ed il conseguente scoppio di "bolle speculative". Per questo motivo l'applicazione della nuova normativa sarà del tutto evanescente se non accompagnata dall'applicazione del "secondo e del terzo pilastro" di cui è formata. Nessuna normativa sarà in grado di portare i benefici auspicati se non è accompagnata da correttezza e senso profondo dell'etica. La cronaca recente ha mostrato tutte le fragilità del nostro sistema economico, infatti, il danno all'immagine del Paese è stato molto più grande della somma dei singoli scandali finanziari.

\section{Appendice}

In appendice poniamo il coefficiente di solvibilità (CS) di una banca, secondo Basilea2 verrà così calcolato: 
PV

$\mathrm{CS}=$

\section{$A P R C+A P R M+A P R O$}

Dove:

PV = Patrimonio di Vigilanza,

$\mathrm{APRC}=$ Attivo ponderato per il rischio di credito,

$\mathrm{APRM}=$ Attivo ponderato per il rischio di mercato,

$\mathrm{APRO}=$ Attivo ponderato per il rischio operativo.

Secondo la normativa, il CS non può essere inferiore a 0,08.

SISTEMA DI RATING ESTERNI

Rating Standard\&Poor's, Moody's Fitch, DCR

\begin{tabular}{|c|c|c|c|c|}
\hline Moody's & S\&P & Fitch & DCR & Definizione \\
\hline Aaa & AAA & AAA & AAA & $\begin{array}{l}\text { Prime. Massima solvibilità - giudizio } \\
\text { Eccellente }\end{array}$ \\
\hline Aal & $\mathrm{AA}^{+}$ & $\mathrm{AA}+$ & $\mathrm{AA}^{+}$ & \multirow{3}{*}{$\begin{array}{l}\text { High Grade Qualità elevata - giudizio } \\
\text { ottimo }\end{array}$} \\
\hline $\mathrm{Aa} 2$ & AA & AA & $\mathrm{AA}$ & \\
\hline $\mathrm{Aa3}$ & AA- & AA- & AA- & \\
\hline Al & $\mathrm{A}+$ & $\mathrm{A}^{+}$ & $\mathrm{A}+$ & \multirow{3}{*}{$\begin{array}{l}\text { Upper Medium Grade livello medio alto - } \\
\text { giudizio buono. }\end{array}$} \\
\hline $\mathrm{A} 2$ & A & A & $\mathrm{A}$ & \\
\hline $\mathrm{A} 3$ & A- & A- & A- & \\
\hline Baal & $\mathrm{BBB}+$ & BBB + & $\mathrm{BBB}+$ & \multirow{3}{*}{$\begin{array}{l}\text { Lower Medium Grade livello medio basso } \\
\text { - giudizio sufficiente. }\end{array}$} \\
\hline $\mathrm{Baa2}$ & BBB & BBB & BBB & \\
\hline $\mathrm{Baa} 3$ & BBB- & BBB- & BBB- & \\
\hline Bal & $\mathrm{BB}+$ & $\mathrm{BB}+$ & $\mathrm{BB}+$ & \multirow[t]{3}{*}{ Categoria speculativa giudizio: discutibile } \\
\hline $\mathrm{Ba} 2$ & BB & BB & $\mathrm{BB}$ & \\
\hline $\mathrm{Ba} 3$ & BB- & BB- & BB- & \\
\hline $\mathrm{Bl}$ & $\mathrm{B}+$ & $\mathrm{B}+$ & $\mathrm{B}+$ & \multirow{3}{*}{$\begin{array}{l}\text { Highly Speculative livello basso - titoli } \\
\text { altamente speculativi - giudizio scarso. }\end{array}$} \\
\hline B2 & B & B & B & \\
\hline B3 & B- & B- & B- & \\
\hline $\mathrm{Caa}$ & $\mathrm{CCC}+$ & $\mathrm{CCC}$ & $\mathrm{CCC}$ & \multirow{2}{*}{$\begin{array}{l}\text { :Substantial Risk livello basso - possibile } \\
\text { inadempimento - giudizio molto scarso. }\end{array}$} \\
\hline & CCC CCC- & & & \\
\hline $\mathrm{Ca}$ & $\mathrm{CC}$ & & & $\begin{array}{l}\text { In Poor Standing titoli altamente } \\
\text { speculativi - giudizio estremamente scarso }\end{array}$ \\
\hline \multirow[t]{5}{*}{ C } & $\mathrm{C}$ & & & $\begin{array}{l}\text { Possibilità di Default inadempienza } \\
\text { emittente altamente probabile. Evidente } \\
\text { crisi di liquidità }\end{array}$ \\
\hline & & DDD & & \multirow{4}{*}{$\begin{array}{l}\text { Default Emittente: inadempiente. } \\
\text { Recupero del capitale investito altamente } \\
\text { improbabile }\end{array}$} \\
\hline & & DD & DD & \\
\hline & D & D & & \\
\hline & & & DP & \\
\hline
\end{tabular}


Questi dati tecnici omessi nelle parti principali dello studio potrebbero essere rilevanti da un punto di vista di mero calcolo da parte di un creditore o della stessa banca che vuole avere accesso al suo coefficiente di solvibilità.

\section{Bibliografia}

AKERLOF G.A., Intervista a Radio 24, Milano, 10 ottobre 2001;

BANCA DEI REGOLAMENTI INTERNAZIONALI, (2003), Convergenza internazionale della misurazione del capitale e dei Coefficienti patrimoniali, Basilea;

BARAVELLI M., (2003), Strategia e organizzazione della banca, EGEA, Milano; COMITATO DI BASILEA 2006, PAR. V;

DE CRISTOFANO N., (2005), Le nuove opportunità professionali per il commercialista, Catania, manoscritto;

GOMPERS P., (1995) Optimal Investment, Monitoring and the Staging of Venture Capital, Journal of Finance, 50;

JENSEN M.C. E W. H. MECKLING, (1976), Theory of the Firm: Managerial Behavior, Agency Costs and Ownership Structure, "Journal of Financial Economics", III, n4;

LOCATELLI R., MAGISTRETTI E., SCALERANDI P. E G. CAROSIO, (2001), II rischio operativo, Quaderno n. 193, Università Cattolica del Sacro CuoreFacoltà di Scienze Bancarie Finanziarie e Assicurative, Roma;

METELLI F., (2005), Basilea2 che cosa cambia, II Sole 24 ore libri, $2^{\circ}$ edizione, Milano;

MOSCATO D., (2005), Le nuove opportunità professionali per il commercialista, Catania, manoscritto;

NELSON R.R., (1959), The Simple Economics of Basic Scientific Research, "Journal of Political Economy", LXVII, n 3 ;

ONADO M., (2004), La banca come impresa, il Mulino, Bologna;

ORIANI R., (2004), Innovazione tecnologica, valore economico e mercati finanziari, il Mulino, Bologna;

PARISI S., (2005), Le opportunità per le banche, le imprese ed i professionisti, Catania, manoscritto;

PIOL E., (2004), II sogno di un'impresa, II Sole 24Ore, Milano; 
PISTELLI F., (2001), Misurare e gestire il rischio di credito nelle banche: una guida metodologica, Fondo Interbancario di Tutela dei Depositi, Roma;

RAMPINI F., (2000), New Economy, Laterza, Bari;

RESTI A., (2001), Misurare e gestire il rischio di credito nelle banche: una guida metodologica, Fondo Interbancario di Tutela dei Depositi, Roma;

RESTI A., (2003), Una Guida a Basilea2, Direzione Risk Management Banca Intesa, Milano;

SASSI S., (1940), II sistema dei rischi d'impresa, Vallardi, Milano.

\section{Sitografia}

www.eurating.it, II rating: che cosa è, (2005);

www.basileadue.it, Cosa cambia per le imprese; (2006);

www.miranet.it/dossier/strumenti_finanziamento_alternativi_basilea2.html, 2006;

http://agevolazionifinanziarie.consrag.it/forum/rating-prenditore.asp;

http://www.sella.it/gbs/trading/formazione/glossario/include/b/29_corpo.jsp; 